British Journal of Ophthalmology, 1981, 65, 97-100

\title{
Clinical aspects of the measurement of oxygen flux into the cornea
}

\author{
JUDITH MORRIS AND MONTAGUE RUBEN \\ From Moorfields Eye Hospital, City Road, London ECIV 2PD
}

SUMmaRY A comparison was firstly done of the oxygen flux through the Bausch and Lomb Plano T lens, the Bausch \& Lomb U3 lens, and the Duragel 75 lens with a sensor on the normal eye. The results showed that the Bausch and Lomb Plano $T$ lens was the best for use with the sensor probe. The sensor was then used to obtain a comparison with the normal oxygen flux through a therapeutic lens on keratoplastic eyes and eyes with bullous keratopathy. The results showed that both conditions had a lower oxygen consumption than normal and that eyes with bullous keratopathy predictably had the lowest value.

The soft lens, when larger than the corneal diameter, acts as an occlusive membrane. The manner in which it prevents water and gas movement have been extensively investigated over the past decade. ${ }^{1-3}$ The relationship between gas movement and the permeability of the lens material has also been investigated. The workers in this field of study recognise that lens form, particularly thickness, temperature, water content, and the physicochemical properties of the plastic determine the gas transmissibility of the lens.

The health of the corneal epithelium and stroma depend on a continually functioning metabolic process by these living tissues. The corneal rate of take up of oxygen measured by means of a sensor probe must be related to oxygen diffusion in the corneal tissues surrounding the sensor probe. Therefore one would expect in the 2 groups of eyes, one normal and the other abnormal, results related to the degree of abnormality.

In almost all cases of keratoplasty ${ }^{4}$ some degree of stromal oedema and endothelial decreased density is present. Consequently, are the results obtained related to a larger stromal 'pool' of oxygen because of greater corneal thickness? The oxygen tension is likely to be low in these abnormally thick stromal zones. The same argument would apply to bullous keratopathy, but here the presence of an abnormal devitalised epithelium may show a decreased oxygen requirement. Furthermore, in both instances the presence of corneal blood vessels would provide a

Correspondence to Mr M. Ruben. corneal zone of high oxygen tension, and so one would expect a slow oxygen take-up from the soft lens (when in situ) by the sensor probe. In any event can a technique that applies the sensor to a soft contact lens worn on the eye be of value in evaluating the therapeutic lens on the eye and thus its likelihood of causing gas deficiency problems? A comparison between the objective results obtained with a lens in situ on normal eyes known to survive long periods of such contact lens wear with the abnormal eye will be of value.

We therefore had 2 objectives: (1) the comparison of the oxygen flux through the Bausch and Lomb Plano T lens, the Bausch and Lomb Type U3, and the Duragel 75 therapeutic lens on the normal eye; (2) the comparison of the oxygen flux through a therapeutic soft lens on $(a)$ keratoplastic human eyes and $(b)$ bullous keratopathic human eyes with that of normal eyes.

\section{Materials and methods}

The experimental procedure involved applying a polarographic oxygen sensor to a soft contact lens on the eye and recording the decrease in oxygen content of the lens as the cornea consumed the oxygen stored in the lens. The sensor when on the eye provided the only available significant source of oxygen, so the rate of oxygen depletion of the lens was a measure of corneal oxygen consumptionthe tear film was ignored; its oxygen store as a fraction of the whole was small.

The oxygen probe used was the Radiometer Sensor Model E5047/0, which consists of a platinum 
cathode and a silver anode embedded in a plastic carrier. The available oxygen was reduced at the platinum cathode and the resulting current measured by an adapted Schema Versatae Amplifier with a read-out on a Heathkit servorecorder at a speed of $5 \mathrm{~s} /$ inch $(2 \mathrm{~s} / \mathrm{cm})$.

A polypropylene membrane served to maintain a small amount of electrolyte solution in contact with the anode and cathode of the probe. The sensor itself was kept at a constant temperature of $38^{\circ} \mathrm{C}$ in an aerated saline bath.

The sensor was calibrated at $35^{\circ} \mathrm{C}$ by first placing it in air-saturated saline and then in a solution of alkaline sulphite. The latter has zero oxygen tension. The recorder level from these 2 solutions was considered to be $155 \mathrm{mmHg}$ and zero oxygen tension respectively. This span of oxygen tension was then used to convert the records obtained when the sensor was on the contact lens to oxygen tension values.

The distribution of oxygen tension in the lens after the sensor is applied has been calculated by I. Fatt ${ }^{5}$ on the basis of an analysis by Carslaw and Jaeger. ${ }^{6}$ The calculation requires $\mathrm{D}$, the diffusion coefficient of oxygen in the lens material; $\mathrm{k}$, the solubility of oxygen in the contact lens material; and $L$, the lens thickness. The steady state flux of oxygen through the contact lens and into the cornea before the sensor is applied is given by:

$$
\frac{J}{A}=\frac{D k}{L}\left(P_{a}-P_{1}\right)
$$

where $\mathbf{J} / \mathbf{A}$ is the number of millilitres of oxygen going into the cornea per second per square centimetre of corneal area. We can calculate the steady state oxygen flux, $\mathrm{J} / \mathrm{A}$, into the cornea under a soft contact lens by measuring the slope of the oxygen tension versus time record as obtained from a sensor pressed on to the contact lens.

To calculate from the data obtained various columns have to be set up (Table 1): Column 1 is the time in seconds that has elapsed since the sensor was applied to the lens. Column 2 is the reading on the recorder paper. Column 3 is the corrected reading from the recorder paper. Column 4 gives the oxygen tension converted to a percentage of the value at zero time. Column 5 is the recorder reading converted to $\mathrm{mmHg}$ oxygen tension. This column is for the construction of a logarithmic plot of recorded oxygen tension as a function of time. Column 6 is the logarithm value of the $\mathrm{PO}_{2}$ value from column 5 .

The slope of the oxygen tension versus time curve, needed to calculate the flux into the cornea, can be calculated from the graphical data. The plot of logarithm of oxygen tension versus time is a straight line, so the differential $\mathrm{dp} / \mathrm{dt}$ can be calculated in column 7 from $\mathrm{dp} / \mathrm{dt}=\mathbf{P}_{\mathbf{0}} \cdot \mathrm{d} \log \mathrm{Po}_{2} / \mathrm{dt}$ where $\mathrm{d} \log$ $\mathrm{Po}_{2}$ is the difference in logarithm of $\mathrm{Po}_{2}$ for 2 points on the line differing by a time interval dt.

Column 8 is the data calculated by multiplying the time points from column 1 by $D / L^{2}$. The value of $D$, the diffusion coefficient of the lens, was obtained from a paper by I. Fatt, ${ }^{5}$ and $L$ of the lens was measured on an electrical thickness gauge. Column 9 is from a term that can be obtained from the $\mathrm{Dt} / \mathrm{L}^{2}$ values from a specified graph. ${ }^{5}$ Column 10 is the tabulation of the numbers in column 9 multiplied, row by row, by $\mathrm{dp} / \mathrm{dt}$ from column 7 and by $\mathrm{kL}$. The numbers in column 10 are the oxygen flux into the cornea stated as $\mathrm{ml} \mathrm{O}_{2}$ (STP)/ $\mathrm{cm}^{2} \times$ s. (STP $=$ standard temperature and pressure.) Column 11 gives a more commonly used unit for flux, $\mu 1 \mathrm{O}_{2}$ (STP) $/ \mathrm{cm}^{2} \mathrm{~h}$ which is obtained from column 10.

\section{Results}

Firstly the normal subjects were used. The lenses chosen for comparison were the Bausch and Lomb Plano T, the Bausch and Lomb U3 (38\% water content), and the Duragel 75 ( $75 \%$ water content). In each case the lenses were fitted to the same subject on the same day and were worn for several minutes before the polarographic oxygen sensor was applied.

After the lens was comfortable on the subject's eye the lid was held open for a few seconds. Then the sensor was taken from the air-saturated saline bath at $38^{\circ} \mathrm{C}$ and when pressed on to the lens was approximately at $35^{\circ} \mathrm{C}$. The effects of blinking on oxygen tension distribution in the lens are partially eliminated by holding the lids open for a few seconds before the sensor contact.

From the tabulated results for the Duragel 75 , $D$ at $35^{\circ} \mathrm{C}$ will be about $1 \times 10^{-5} \mathrm{~cm} / \mathrm{s} .{ }^{5}$ If the lens is from 0.15 to $0.20 \mathrm{~mm}$ thick, then the group $\mathrm{Dt} / \mathrm{L}^{2}$

Table 1 Data columns for calculation

\begin{tabular}{lllllllllll}
\hline$(1)$ & $(2)$ & $(3)$ & $(4)$ & $(5)$ & $(6)$ & $(7)$ & $(8)$ & $(9)$ & $(10)$ & $(11)$ \\
\hline Time & $\begin{array}{l}\text { Gross } \\
\text { rec. }\end{array}$ & $\begin{array}{l}\mathrm{Net} \\
\text { rec. }\end{array}$ & $\% \mathrm{Po}_{2}$ & $\mathrm{Po}_{2}$ & $\begin{array}{l}\log \\
\mathrm{Po}_{2}\end{array}$ & P. $\frac{\mathrm{d} \cdot \log \mathrm{Po}_{2}}{\mathrm{dt}}=\frac{\mathrm{dP}}{\mathrm{dt}}$ & $\frac{\mathrm{Dt}}{\mathrm{L}^{2}}$ & $\frac{1}{2 \mathrm{E}}$ & $\frac{\mathrm{J}}{\mathrm{A}}=\frac{\mathrm{k} 1 \mathrm{dp}}{2 \mathrm{E} \mathrm{dt}}$ & $\frac{\mathrm{J}}{\mathrm{A}}$ \\
\hline
\end{tabular}


becomes greater than 0.1 at times greater than 4 seconds. The results also show a continually increasing flux into the eye instead of levelling off after 10 seconds. This was because the effect of the sensor through the Duragel 75 lens was felt at the cornea in 5 seconds and the mathematical procedure then becomes invalid. This agreed with work done by Fatt ${ }^{5}$ on Sauflon 85 lenses.

The Bausch and Lomb U3 lenses of thickness $\mathbf{L}=\mathbf{0 . 0 8}$ also gave the same pattern of results as the Duragel 75. Presumably their problem was the thinness of the lenses as opposed to the water content of the Duragel material.

Once it had been ascertained that the lens of use was going to be the Bausch and Lomb Plano T lens, the reproducibility of the results with this lens was tested. By applying the sensor 10 times to the same lens on the same eye with a few minutes' gap between each application and working through the results of each application, it was found that for a mean value of $\mathrm{J} / \mathrm{A}$ equal to $3.54 \mu 1 \mathrm{O}_{2}$ (STP) $/ \mathrm{cm}^{2} \mathrm{~h}$ the standard deviation was 0.22 and a reasonable graph was obtained.

Twelve normal eyes were then measured with a standard Plano $T$ lens and the mean value for $J / A$ was found to be $3.40 \mu 1 \mathrm{O}_{2}$ (STP) $/ \mathrm{cm}^{2} \mathrm{~h}$. The lowest value was: $2.54 \mu 1 \mathrm{O}_{2}$ (STP) $/ \mathrm{cm}^{2} \mathrm{~h}$ and the highest: $3.80 \mu 1 \mathrm{O}_{2}(\mathrm{STP}) \mathrm{cm}^{2} \mathrm{~h}, \mathrm{SD}=\mathbf{0} \cdot 35$.

Pachometry was not carried out on these subjects, as it was assumed their thicknesses were within normal limits.

The keratoplastic eyes were then studied. The patients chosen were those who were not wearing a contact lens on the keratoplastic eye, so that their oxygen need could be compared with that of the normal noncontact lens wearing eyes. Twelve cases were studied. Their diagnosis was: keratoconus, 7 cases; herpes simplex keratitis, 1 case; neurotrophic keratitis, 1 case; Fuchs's dystrophy, 1 case; bullous keratopathy, 1 case; dendritic ulcer, 1 case. The state of the graft and host was examined before their oxygen flow was measured as was their graft thickness (Table 2). In only a few eyes was there mild or moderate graft scarring, while there were 4 cases with mild or moderate new vessels in host or graft tissue. Out of the 12 eyes 3 had a history of graft rejection, 2 of which were cases with mild or moderate new vessel changes.

The mean of the oxygen flux results for the 12 graft eyes was $J / A=2.76 \mu 1 \mathrm{O}_{2}(\mathrm{STP}) / \mathrm{cm}^{2} \mathrm{~h}$. This shows a lower oxygen need to maintain adequate tissue function than the normal. The SD $=0.37$.

If we relate individual results to the state of their eyes, it is found that the 3 cases with a history of graft rejection have the lowest oxygen flux measurements: $2 \cdot 28 \mu 1 \mathrm{O}_{2}$ (STP) $/ \mathrm{cm}^{2} \mathrm{~h}, 2 \cdot 35 \mu 1 \mathrm{O}_{2}$ (STP) $/ \mathrm{cm}^{2}$
Table 2 State of graft epithelium and stroma

\begin{tabular}{llrllll}
\hline & & \multicolumn{3}{c}{ None } & Mild & \multicolumn{2}{c}{ ate } & Severe \\
\hline Graft scarring: & Bowman's & 9 & 1 & 2 & - \\
& Sub-Bowman's & 10 & 2 & - & - \\
& Stromal & 9 & 1 & 2 & - \\
& Endothelial & 10 & 1 & 1 & - \\
New vessels: & Superficial-host & 8 & 1 & 3 & - \\
& & graft & 11 & 1 & - & - \\
& Deep & host & 10 & 1 & - & - \\
& \multicolumn{1}{c}{ graft } & 11 & 1 & - & - \\
& Uveitis-old quiescent & 9 & 2 & - & 1 \\
& \multicolumn{2}{c}{ active } & 12 & - & - & - \\
\hline
\end{tabular}

h, and $2.44 \mu 1 \mathrm{O}_{2}$ (STP) $/ \mathrm{cm}^{2}$ h. Two of these cases had new vessel involvement at the superficial and deep level for the host and graft tissue. Of the other 2 cases with new vessel involvement, one had superficial ones related to loose sutures, while the other with moderate superficial vessels had an oxygen flux of $3.00 \mu 1 \mathrm{O}_{2}$ (STP) $/ \mathrm{cm}^{2} \mathrm{~h}$.

On trying to relate corneal thickness measurements with oxygen flux we did not find any definite relationship.

Only 4 bullous keratopathic eyes were studied. It was difficult to use acute eyes as the experimental method was not applicable to a painful eye. Therefore eyes of the chronic type were studied, and a mean flux $\mathrm{J} / \mathrm{A}=2 \cdot 30 \mu 1 \mathrm{O}_{2}(\mathrm{STP}) / \mathrm{cm}^{2} \mathrm{~h}$ was achieved, standard deviation $=0 \cdot 19$.

\section{Discussion}

The results for the normal eyes show a slightly lower value than has been reported, but this may be a consequence of instrumentation. ${ }^{5}$ However, the results were in a reasonably reproducible range and did not show great personal variation for the 12 normal persons studied.

So far as the keratoplastic eyes are concerned, less oxygen was needed to maintain functions. This was especially so for the eyes which had new vessel involvement. It appears from the results that there may be a supply of oxygen from these vessels to the graft tissue such that the graft tissue needs less atmospheric oxygen to maintain its function.

For the 3 eyes which had a history of graft rejection and showed a low oxygen flux we have by the Mann-Whitney $U$ test $95 \%$ confidence that there is a difference between them and the rest of the keratoplastic eyes. For the bullous keratopathic eyes the epithelium is to all intents and purposes defunct. This is shown by the low values of oxygen 
flux obtained. For both the keratoplastic and bullous keratopathic eyes the differences in means were more than twice the pooled standard deviations in relation to the normal persons, so we have $95 \%$ confidence that the difference is real.

\section{Conclusions}

This work and results show that the Bausch and Lomb Plano T lens can be used as a standard for oxygen studies. Its application in normal and abnormal eyes makes it possible to gain information on the viability of the epithelium. The intention is to follow up this study with further work on the aphakic eye.
We thank Dr I. Fatt for his advice and assistance in preparing this paper.

\section{References}

1 Fatt I, Bieber M. Steady state of oxygen and carbon dioxide in vivo of open and closed eye. Exp Eye Res 1968; 7: 103-12.

2 Dreyfus M. Development of the hydrophilic contact lens. Contactologia 1980; 2: 226-35.

3 Kinsey VE. Explanation of corneal haze. Am J Ophthalmol 1952; 35: 691-5.

4 Ruben M, Colebrook E, Guillon M. Keratoconus and keratoplasty thickness. Br J Ophthalmol 1979; 63: 790-3.

5 Fatt I. Measurement of oxygen flux into the cornea by pressing a sensor on to a soft contact lens on the eye. Am J Optometry 1978; 55: 294-301.

6 Carslaw H, Jaeger JC. Heat Conduction in Solids. Oxford: Oxford University Press, 1959. 